\title{
Ionic Conductivity and Mobility of Poly(propylene oxide) Networks Dissolving Alkali Metal Thiocyanates
}

\author{
Masayoshi Watanabe, Kohei Sanui, Naoya OGata, \\ Fumio INOUE, ${ }^{*}$ Tadahiko KobaYASHI, ${ }^{*}$ \\ and Zentaro OHTAKI* \\ Department of Chemistry, Sophia University, 7-1 Kioi-cho, \\ Chiyoda-ku, Tokyo 102, Japan \\ *Department of Electrical and Electronic Engineering, Sophia University, \\ 7-1 Kioi-cho, Chiyoda-ku, Tokyo 102, Japan
}

(Received April 6, 1984)

\begin{abstract}
Ionic conducitivity and mobility were investigated in poly(propylene oxide) (PPO) networks dissolving the alkali metal thiocyanates, LiSCN, NaSCN, and KSCN. The temperature dependence of ionic conductivity of the PPO complexes, estimated from complex impedance method, obeyed the WLF-type equation with $C_{1}=9-15$ and $C_{2}=35-80$. At a reduced temperature of $T_{\mathrm{g}}+100 \mathrm{C}$, the magnitude of conductivity was of the order of $10^{-6}-10^{-5} \mathrm{Scm}^{-1}$, following the order of PPO-KSCN $>$ PPO-NaSCN $>$ PPO-LiSCN. Ionic mobility was estimated from an initial slope of the current-time curve with a first d.c. voltage application and from the time of current maximum after reversal of the applied voltage polarity, assuming the clean-up effect of carrier ions to dominate the time dependence of the current. The ionic mobility at $T_{\mathrm{g}}+100 \mathrm{C}$ did not greatly depend on the kinds of incorporated salts and was of the order of $10^{-6}$ $10^{-5} \mathrm{~cm}^{2} \mathrm{~V}^{-1} \mathrm{~s}^{-1}$. Somewhat lower ionic mobilities were deduced by the latter method.

KEY WORDS Ionic Conductor / Poly(propylene oxide) / Complex Impedance Method / Ionic Conductivity / WLF-Type Equation / Ionic Mobility /
\end{abstract}

An exciting new direction iri the research of fast ionic conductors is aimed at ionic conductivity in polymers. ${ }^{1-13}$ Most of the research in this field has been concerned with polymer complexes formed by alkali metal salts and polyethers such as poly(ethylene oxide) $(\mathrm{PEO})^{1-9}$ and poly(propylene oxide) (PPO $)^{2.6 .10-13}$ Certain complexes show a relatively high ionic conductivity of $10^{-5}-10^{-4}$ $\mathrm{S} \mathrm{cm}^{-1}$ at moderate temperatures. The ionic conductivity is influenced by many factors such as chemical structure of polyethers, ${ }^{2.6 .10}$ incorporated salt species. ${ }^{1-3.10}$ and degree of crystallinity. ${ }^{2.4 .5}$ Conductivity is determined by both the number of carrier ions and their mobility, which may thus be influenced by these factors. However, hardly anything is known about carrier generation and migration in the polyether complexes.

In this study, an investigation was made of the conductivity and mobility of polyether complexes. PEO has been shown to favor the formation of crystalline complexes with certain kinds of alkali metal salts ${ }^{1-5}$ and whether or not PEO forms a crystalline complex depends on the kind of salt. ${ }^{2}$ In contrast, PPO forms amorphous complexes with alkali metal salts. ${ }^{2.10-13}$ To simplify the discussion of ionic conductivity and mobility, amorphous PPO networks were selected as host polymers for alkali metal thiothianates (LiSCN, NaSCN, and $\mathrm{KSCN}$ ) in this study. The temperature dependence of the ionic conductivity of the PPO complexes obeyed the WLF-type equa- 
tion with similar $\mathrm{C}_{1}$ and $\mathrm{C}_{2}$ values when glass transition temperarture $\left(T_{\mathrm{g}}\right)$ was used as a standard temperature. Ionic mobility was estimated from an initial slope of the current-time curve with the first d.c. voltage application and from the time of current maximum after reversal of the applied voltage polarity. The difference in ionic conductivity depending on incorporated salts at a reduced temperature of $T_{\mathrm{g}}+100^{\circ} \mathrm{C}$ is discussed in terms of carrier generation and migration.

\section{EXPERIMENTAL}

\section{Samples}

PPO networks were prepared by a crosslinking reaction of triol-type PPO (mol.wt.= 3000) with tolylene-2,4-diisocyanate. The thickness of the networks was from 0.82 $0.86 \mathrm{~mm}$. The alkali metal salts of LiSCN, $\mathrm{NaSCN}$, and KSCN were dissolved in the networks by dipping them into the acetone solution of each salt, followed by evaporating the solvent completely. The precise conditions for the preparation of the samples are described elsewhere. ${ }^{13}$ The concentration of the salt in the networks was represented by the molar ratio of the salt to the repeating unit of PPO ([Salt]/[PO unit]) and was kept at 0.05 in all samples. This molar ratio corresponds to the weight ratios (salt to PPO networks) of 0.051, 0.064, and 0.077 for the PPO-LiSCN, PPO-NaSCN, and PPO-KSCN complexes, respectively.

\section{Methods}

A disk-like sample (13 $\mathrm{mm}$ in diameter), sandwiched between platinum electrodes, was packed in a sealed cell with stainless steel terminals in contact with the measuring device. Impedance measurements were made on the cell with a Hewlett-Packard 4800 A vector impedance meter equipped with a Takeda Riken TR5821 electronic counter over a frequency range of $5 \mathrm{~Hz}-500 \mathrm{kHz}$. The oscilation levels were $2.7 \mathrm{mV}, 27 \mathrm{mV}, 270 \mathrm{mV}$, and $2.7 \mathrm{~V}$ depending on the sample impedance ranges of $1-10 \mathrm{k} \Omega, 10-100 \mathrm{k} \Omega, 0.1-1 \mathrm{M} \Omega$, and $1-$ $10 \mathrm{M} \Omega$, respectively. In this range of oscillation, the impedance responce was nearly linear. D.c. measurements were carried out using a Keithley $610 \mathrm{C}$ electrometer and a Yokogawa Electric Work 2851 standard power supply. The applied d.c. voltage was $2.5 \mathrm{~V}$. A well-shielded thermostated oven containing cell systems was used for temperature regulation.

Differential scanning calorimetry (DSC) was carried out using a Rigaku Denki 8085 DSC apparatus at a heating rate of $20 \mathrm{C} \mathrm{min}^{-1}$. XRay diffraction patterns were measured with a Rigaku Denki RAD-IIA diffractometer. The radiation used was $\mathrm{Ni}$-filtered $\mathrm{Cu} K_{\alpha}$.

\section{RESULTS AND DISCUSSION}

\section{Temperature Dependence of Ionic Conductivity}

The equivalent circuit shown in Figure 1 was used for interpreting the complex impedance diagrams of the PPO-alkali metal thiocyanate complexes in contact with platinum electrodes. Figure 2 shows the complex impedance diagrams of the PPO-KSCN complex at various temperatures. In the figure, the horizontal axis is the resistance $\left(Z^{\prime}\right)$, i.e., the real part of the impedance, and the vertical axis is the reactance $\left(Z^{\prime \prime}\right)$, i.e., the imaginary part of the impedance. The measured plots were considered to be located on a part of the diagram that is a high frequency arc with a low frequency branch. Since $C_{\mathrm{e}}$ is generally larger

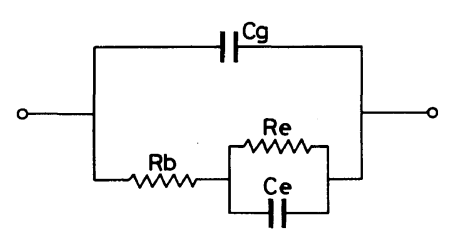

Figure 1. Equivalent circuit for interpreting the complex impedance diagram. $R_{\mathrm{b}}$, bulk electrolyte resistance; $C_{\mathrm{g}}$, geometrical capacitance; $R_{\mathrm{e}}$, electrolyte/electrode interfacial resistance; $C_{\mathrm{e}}$, electrolyte/electrode interfacial capacitance. 

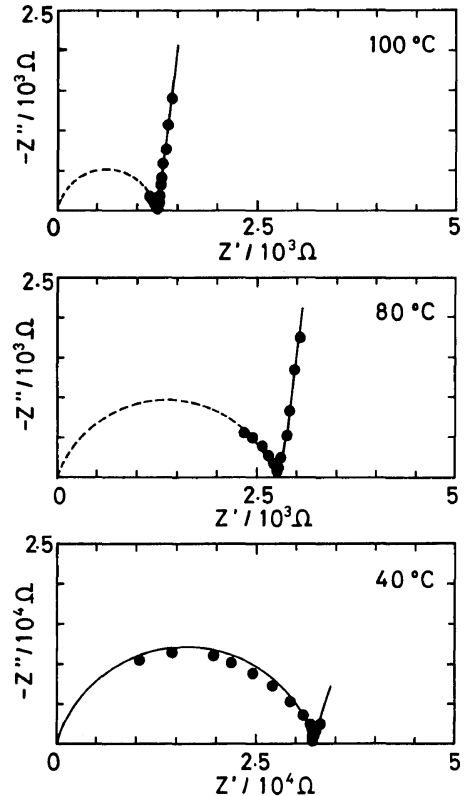

Figure 2. Complex impedance diagrams for the PPO$\mathrm{KSCN}$ complex $0.82 \mathrm{~mm}$ in thickness, in contact with platinum electrodes.

than $C_{\mathrm{g}}$ by several orders of magnitude, the low frequency branches corresponding to the interfacial impedance were interpreted by the equivalent circuit of $R_{\mathrm{b}}$ in series with parallel combination of $R_{\mathrm{e}}$ and $C_{\mathrm{e}} \cdot{ }^{13}$ Thus, the branches seemed to be a high frequency part of another large arc. The high frequency arc in Figure 2 corresponds to the bulk impedance expressed by the equivalent circuit of the parallel combination of $R_{\mathrm{b}}$ and $C_{\mathrm{g}} \cdot{ }^{13}$ High frequency ends of the branches and low frequency ends of the arcs coincide with $R_{\mathrm{b}}$. The $R_{\mathrm{b}}$ values increased greatly with decreasing temperature. Figure 3 shows the complex impedance diagrams of the PPO-KSCN, PPONaSCN, and PPO-LiSCN complexes at $60^{\circ} \mathrm{C}$. The $R_{\mathrm{b}}$ value of each PPO complex could be estimated from the diagrams. From the low frequency branches, the arc expressing the interfacial impedance was considered to be considerably larger than that expressing the bulk impedance. Thus, platinum seemed to act as ion-blocking electrodes, and the charge
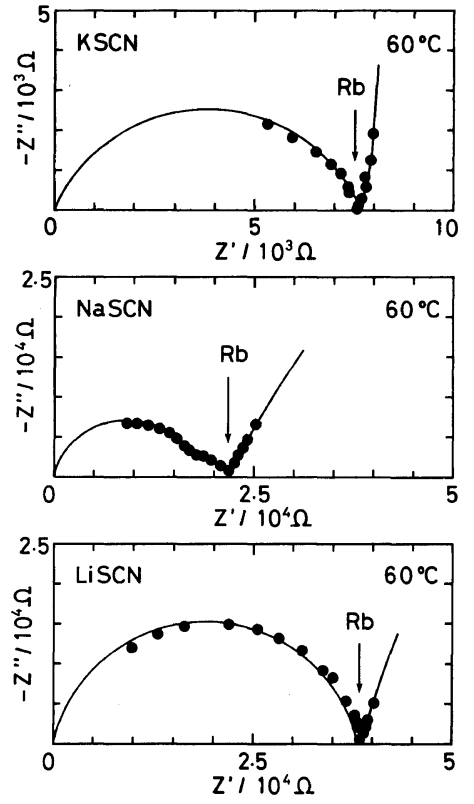

Figure 3. Complex impedance diagrams of PPO complexes in contact with platinum electrodes at $60^{\circ} \mathrm{C}$. Sample thickness: PPO-KSCN, $0.82 \mathrm{~mm}$; PPO-NaSCN, $0.84 \mathrm{~mm}$; PPO-LiSCN, $0.86 \mathrm{~mm}$.

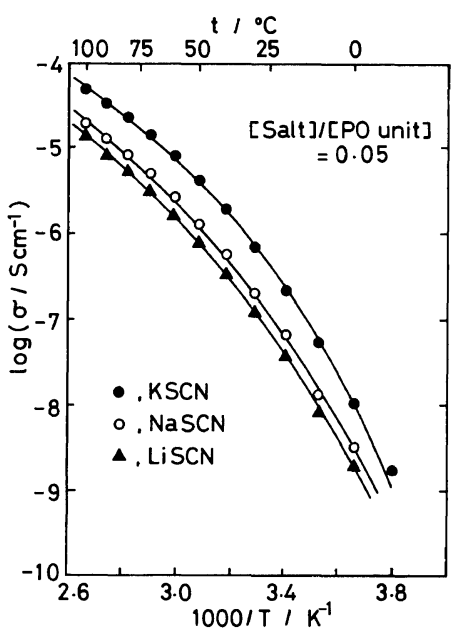

Figure 4. Relation between logarithm of ionic conductivity and reciprocal of absolute temperature.

transfer between the complex and electrodes might occur with great difficulty (large $R_{\mathrm{e}}$ ).

Figure 4 shows the temperature dependence of the ionic conductivity $(\sigma)$ calculated from $R_{\mathrm{b}}$. The $\sigma$ values at the same temperature were 
dependent on the incorporated salt species, and followed the order of PPO-KSCN $>$ PPONaSCN $>$ PPO-LiSCN. The profile of temperature dependence was not straight but a bended curve. DSC measurements of the PPO complexes revealed that no endothermal peaks attributable to melting of microcrystallites existed in the temperature range of -150 to $200^{\circ} \mathrm{C}$. Furthermore, only halos were observed in the X-ray diffraction patterns $(2 \theta=5-$ $40^{\circ} \mathrm{C}$ ) of these complexes. Thus, the PPO complexes were completely amorphous. Since their $T_{\mathrm{g}}$ 's determined from DSC were located below the measuring temperature range, it was expected that the temperature dependence of $\sigma$ was related to that of the segmental mobility of the PPO main chain. Thus, the following WLF-type equation ${ }^{14}$ was used to interpret the temperature dependence of $\sigma$.

$$
\log \frac{\sigma(T)}{\sigma\left(T_{\mathrm{g}}\right)}=\frac{\mathrm{C}_{1}\left(T-T_{\mathrm{g}}\right)}{\mathrm{C}_{2}+\left(T-T_{\mathrm{g}}\right)}
$$

The data sets of $\mathrm{C}_{1}, \mathrm{C}_{2}$, and $\sigma\left(T_{\mathrm{g}}\right)$ were calculated so as to best linearlize the correlation between $1 /\left(T-T_{\mathrm{g}}\right)$ and $1 / \log [\sigma(T) /$ $\left.\sigma\left(T_{\mathrm{g}}\right)\right]$. Since the glass transition zone was observed in DSC charts as a heat capacity jumps with a certain temperature width, the onset and end temperatures in addition to the

Table I. WLF parameters for PPO complexes

\begin{tabular}{|c|c|c|c|c|c|}
\hline \multirow{2}{*}{ Salt } & \multicolumn{2}{|c|}{$T_{\mathrm{g}}^{\mathrm{a}}$} & \multirow{2}{*}{$\frac{\mathrm{C}_{1}}{\mathrm{C}}$} & \multirow{2}{*}{$\frac{\mathrm{C}_{2}}{\mathrm{C}}$} & \multirow{2}{*}{$\frac{\sigma\left(T_{\mathrm{g}}\right)}{\mathrm{Scm}^{-1}}$} \\
\hline & $\mathrm{C}$ & & & & \\
\hline \multirow[t]{3}{*}{ LiSCN } & Center & -33 & 11.7 & 59.9 & $1.2 \times 10^{-13}$ \\
\hline & Onset & -47 & 15.2 & 45.6 & $3.7 \times 10^{-17}$ \\
\hline & End & -19 & 9.5 & 76.7 & $2.4 \times 10^{-11}$ \\
\hline \multirow[t]{3}{*}{$\mathrm{NaSCN}$} & Center & -26 & 10.2 & 62.5 & $3.1 \times 10^{-12}$ \\
\hline & Onset & -38 & 12.9 & 48.5 & $6.0 \times 10^{-15}$ \\
\hline & End & -13 & 8.7 & 80.5 & $1.9 \times 10^{-10}$ \\
\hline \multirow[t]{3}{*}{$\mathrm{KSCN}$} & Center & -30 & 10.8 & 44.8 & $5.1 \times 10^{-13}$ \\
\hline & Onset & -39 & 13.7 & 34.9 & $5.3 \times 10^{-16}$ \\
\hline & End & -19 & 8.7 & 56.5 & $6.2 \times 10^{-11}$ \\
\hline
\end{tabular}

a Center, onset, and end mean center, onset, and end temperatures of heat capacity jumps observed in the glass transition zone of DSC measurements. center temperature of the heat capacity jump were also used in the calculation. Table I shows the WLF parameters obtained. The temperature dependence of $\sigma$ fitted very well the WLF-type equation, and the $\mathrm{C}_{1}$ and $\mathrm{C}_{2}$ values were considered to be comparable to the universal values ${ }^{14}$ of $\mathrm{C}_{1}=17.4$ and $\mathrm{C}_{2}=$ 51.6 for the temperature dependence of the segmental mobility. This implies that the temperature dependence of $\sigma$ is mainly determined by the change in ionic mobility which is correlated closely to segmental mobility. Transport of the carrier ions may cooperate with the segmental motion of the PPO main chain.

\section{Estimation of Ionic Mobility}

As mentioned above, $\sigma$ of the PPO complexes was dependent on the kind of incorporated salt. $\sigma$ is represented by:

$$
\sigma=\sum n z e \mu
$$

where $n$ is the number of carrier ions, $z$, valency of the carrier ions, $e$, the elementary electric charge, and $\mu$, ionic mobility. Thus, the different $\sigma$ values arise from the differences in both or either $n$ and/or $\mu$. We tried to estimate $\mu$ from d.c. measurements. Figure 5(a) shows the time dependence of current with a first application of $2.5 \mathrm{~V}$ in one direction within $30 \mathrm{~min}$, and Figure 5(b) shows the current-time curves after reversal of the applied voltage polarity. The switching time for the reversal was less than $0.5 \times 10^{-3} \mathrm{~s}$. Since the temperature dependence of $\sigma$ obeyed the WLF-type equation, measurements were carried out at a constant reduced temperature of $T_{\mathrm{g}}+100^{\circ} \mathrm{C}$ except for the salt free PPO network. The measuring temperatures are indicated in the figure caption. In the case of the salt free PPO network, the current was so low at $T_{\mathrm{g}}+100^{\circ} \mathrm{C}$ that measurement was made at $T_{\mathrm{g}}+120^{\circ} \mathrm{C}$, i.e., at $70^{\circ} \mathrm{C}$. As can be seen from Figure 5(a), the current through the cell of the PPO complexes decreased rapidly within the first $10 \mathrm{~min}$ and continued to do so gradually, reaching a current level at $30 \mathrm{~min}$, less than the initial 

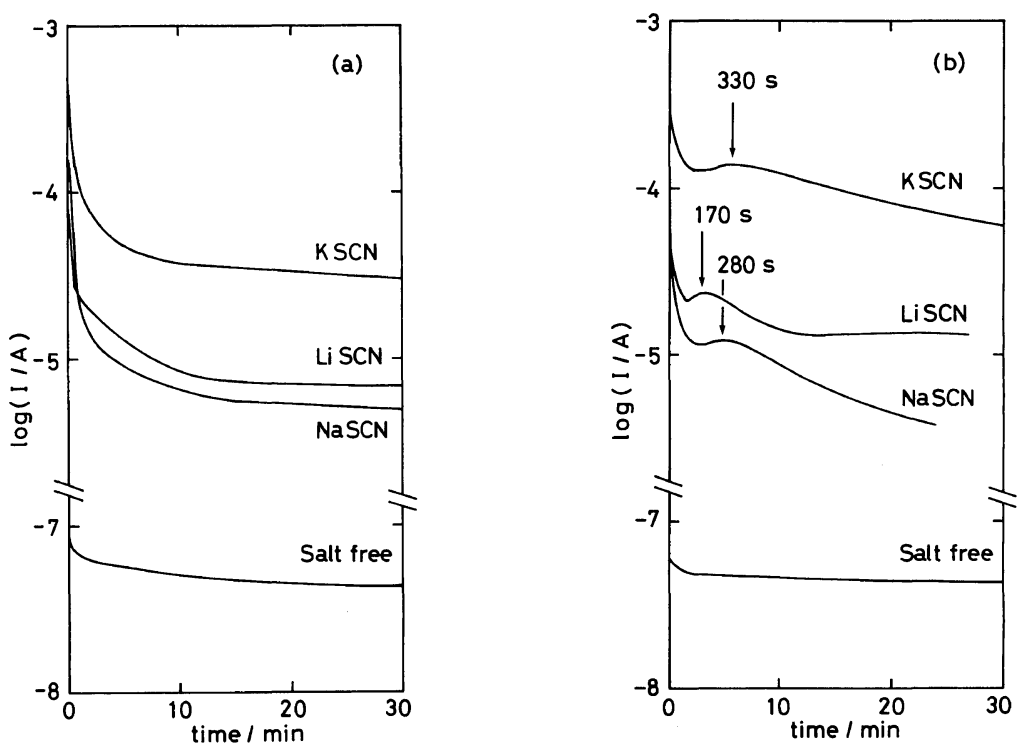

Figure 5. Time dependence of currrent with a first application of d.c. $2.5 \mathrm{~V}$ in one direction, (a); after reversal of the applied voltage polarity, (b). Measurements were carried out at $T_{\mathrm{g}}+100^{\circ} \mathrm{C}$ for PPO complexes and at $T_{\mathrm{g}}+120^{\circ} \mathrm{C}$ for salt free PPO, i.e., at $67^{\circ} \mathrm{C}$ for PPO-LiSCN, at $74^{\circ} \mathrm{C}$ for PPO-NaSCN, at $70^{\circ} \mathrm{C}$ for PPO-KSCN, and at $70^{\circ} \mathrm{C}$ for salt free PPO.

value by one or two order(s) of magnitude. Two contributions are considered the reasons for this decrease: the behavior of the carrier ions and the relaxation of the dipole orientation. In the salt free PPO network the current level was far lower than that in the PPO complexes and the current decrease with time was not so large as for the PPO complexes. We cannot conclude here that the current-time behavior in the salt free PPO network results from the behavior of the carrier ions or dipole orientation relaxation. However, the higher current levels and larger current decreases in the PPO complexes must be due to the incorporation of salts in the PPO networks. That is, the time dependence of the current for the PPO complexes can be explained by carrier ion behavior. Since we used an ion blocking platinum electrode, carrier ions moved in bulk and accumulated in the vicinity of the electrodes with a d.c. voltage application. This accumulation caused a decrease in the number of carrier ions in the bulk (clean-up effect) and a drop in the applied voltage (space charge effect). The current behavior may be explained by these two effects. We can also observe the behavior of carrier ions in Figure 5(b). For the salt free PPO network, the current decreased gradually with time, in a manner similar to that in Figure 5(a). The PPO complexes had a current maximum in the current-time curves at $170 \mathrm{~s}$ for the PPO-LiSCN complex, at $280 \mathrm{~s}$ for the PPONaSCN complex, and at $330 \mathrm{~s}$ for the PPOKSCN complex. The current level at the maximum was higher than that in Figure 5(a) at the same time. These phenomena are correlated to the behavior of the accumulated ion layer in the vicinity of the electrodes during the first application of d.c. voltage.

When the time dependence of current is dominated by the behavior of the carrier ions, the current $(I)$ is expressed by:

$$
I=\frac{S V^{*}}{d} \sum n z e \mu
$$


where $V^{*}$ is the effective voltage across the sample with surface area $(S)$ and thickness $(d)$. In Figure 5(a), the drop in applied voltage may be neglected within the initial short time; that is, $V^{*}$ is assumed to be equal to the applied voltage $(V)$. The initial rapid decrease in current may be caused by a decrease in $n$ in eq 3 , according to Faraday's law. The initial time dependence of current is expressed as ${ }^{15}$

$$
I=I_{\circ} \exp \left(-\mu_{i} V t / d^{2}\right)
$$

where $t$ is time after application of d.c. voltage. Thus, the ionic mobility $\left(\mu_{i}\right)$ may be calculted from the initial slope of the $\log I-t$ curves in Figure 5(a) and eq 4 . The $\mu_{i}$ values obtained are listed in Table II. The current maximum in Figure 5(b) can be explained as follows. ${ }^{15}$ Before reversal of the applied voltage polarity the some carrier ions accumulate in the vicinity of the electrodes. After the reversal, $n$ in the bulk increases owing to release of carrier ions from the accumulated layer. Just after the carrier ions reach the opposite electrodes, the accumulation of carrier ions begins again, resulting in a decrease in $n$. Thus, $n$ in the bulk becomes maximum at $\tau$. The initial decrease in current in Figure 5(b) may be due to carrier ions which did not accumulate during the first polarization. If we assume the average $V^{*}$ in eq 3 during the period of $0-\tau$ to be approximately equal to $V$, the time dependence of the current takes a peak at $\tau$. The ionic mobility $\left(\mu_{\tau}\right)$ may thus be estimated from the following equation:

$$
\tau=\frac{d^{2}}{\mu_{\tau} V}
$$

The $\mu_{\tau}$ values obtained are also listed in Table II. However, these values may be only rough approximations, since agreement of $V^{*}$ with $V$ where the space charge effect is neglected is unlikely. Nevertheless, we consider that ionic mobility by these two methods provides a partial explanation for the carrier migration in the PPO complexes.

As can be seen from Table II, ionic mobility

\begin{tabular}{|c|c|c|c|c|}
\hline \multirow{2}{*}{ Salt } & $T_{\mathrm{g}}^{\mathrm{a}}$ & $\sigma$ & $\mu_{i}$ & $\mu_{\tau}$ \\
\hline & ${ }^{\circ} \mathrm{C}$ & $\mathrm{S} \mathrm{cm}^{-1}$ & $\mathrm{~cm}^{2} \mathrm{~V}^{-1} \mathrm{~s}^{-1}$ & $\mathrm{~cm}^{2} \mathrm{~V}^{-1} \mathrm{~s}^{-1}$ \\
\hline LiSCN & -33 & $3.4 \times 10^{-6}$ & $8.5 \times 10^{-5}$ & $1.7 \times 10^{-5}$ \\
\hline $\mathrm{NaSCN}$ & -26 & $9.3 \times 10^{-6}$ & $4.7 \times 10^{-5}$ & $1.0 \times 10^{-5}$ \\
\hline $\mathrm{KSCN}$ & -30 & $1.5 \times 10^{-5}$ & $7.6 \times 10^{-5}$ & $8.2 \times 10^{-6}$ \\
\hline
\end{tabular}

Table II. Conduction parameters at $T_{\mathrm{g}}+100^{\circ} \mathrm{C}$

a Center temperatures of heat capacity jumps in the glass transition zone of DSC measurements.

reaches $10^{-6}-10^{-5} \mathrm{~cm}^{2} \mathrm{~V}^{-1} \mathrm{~s}^{-1}$ at $T_{\mathrm{g}}$ $+100^{\circ} \mathrm{C}$. The $\mu_{i}$ values are somewhat higher than the $\mu_{\tau}$ values. This may be explained as follows. $\mu_{\tau}$ was calculated using eq 5 . However, the space charge effect causes a drop in the applied voltage during the period of $0-\tau$. Table II also indicates that possibly the difference in ionic mobility depending on salt species is not so large as that in the ionic conductivity. The transference number of ions in the PPO complexes is still not clear at the present. Thus, both cation and anion migrations may contribute to the ionic mobility in this experiment. If the dependence of ionic mobility on the incorporated salt species is considered small, the difference in conductivity may be caused by the number of carrier ions in these three kinds of the PPO complexes.

\section{REFERENCES}

1. P. V. Wright, Br. Polym. J., 7, 319 (1975).

2. M. B. Armand, J. M. Chabagno, and M. J. Duclot, "Fast Ion Transport in Solids," P. Vashishta, J. M. Mundy, and G. K. Shenoy, Ed., North Holland, New York, N.Y., 1979, p 131.

3. R. Dupon, B. L. Papke, M. A. Ratner, D. H. Whitmore, and D. F. Shriver, J. Am. Chem. Soc., 104, 6347 (1982).

4. C. C. Lee and P. V. Wright, Polymer, 23, 681 (1982).

5. D. R. Payne and P. V. Wright, Polymer, 23, 690 (1982).

6. A. Killis, J. F. Le Nest, H. Charadame, and A. Gandini, Makromol. Chem., 183, 2935 (1982).

7. A. Killis, J. F. Le Nest, A. Gandini, and H. Charadame, Macromolecules, 17, 63 (1984).

8. D. F. Shriver, B. L. Papke, M. A. Ratner, R. Dupon, T. Wong, and M. Brodwin, Solid State Ionics, 5, 83 (1981). 
9. E. Tsuchida, H. Ohno, K. Tsunemi, and N. Kobayashi, Solid State Ionics, 11, 227 (1983).

10. M. Watanabe, K. Nagaoka, K. Kanba, and I. Shinohara, Polym. J., 14, 877 (1982).

11. M. Watanabe, J. Ikeda, and I. Shinohara, Polym. J., 15, 65 (1983).

12. M. Watanabe, J. Ikeda, and I. Shinohara, Polym. J.,
15, 175 (1983).

13. M. Watanabe, K. Sanui, N. Ogata, F. Inoue, T. Kobayashi, and Z. Ohtaki, Polym. J., 16, 711 (1984).

14. M. L. Williams, R. F. Landel, and J. D. Ferry, J. Am. Chem. Soc., 77, 3701 (1955).

15. M. Kosaki, H. Ohshima, and M. Ieda, J. Phys. Soc. Jpn., 29, 1012 (1970). 